\title{
Computation and Visualization of Casimir Forces in Arbitrary Geometries: Nonmonotonic Lateral- Wall Forces and the Failure of Proximity-Force Approximations
}

\section{Citation}

Rodriguez, Alejandro, Mihai Ibanescu, Davide lannuzzi, Federico Capasso, J. D. Joannopoulos, and Steven G. Johnson. 2007. "Computation and Visualization of Casimir Forces in Arbitrary Geometries: Nonmonotonic Lateral-Wall Forces and the Failure of Proximity-Force Approximations." Physical Review Letters 99 (8). https://doi.org/10.1103/physrevlett.99.080401.

\section{Permanent link}

http://nrs.harvard.edu/urn-3:HUL.InstRepos:41371573

\section{Terms of Use}

This article was downloaded from Harvard University's DASH repository, and is made available under the terms and conditions applicable to Other Posted Material, as set forth at http:// nrs.harvard.edu/urn-3:HUL.InstRepos:dash.current.terms-of-use\#LAA

\section{Share Your Story}

The Harvard community has made this article openly available.

Please share how this access benefits you. Submit a story.

\section{Accessibility}




\title{
Computation and visualization of Casimir forces in arbitrary geometries: non-monotonic lateral-wall forces and failure of proximity-force approximations
}

\author{
Alejandro Rodriguez, ${ }^{1}$ Mihai Ibanescu, ${ }^{1}$ Davide Iannuzzi, ${ }^{2}$ \\ Federico Capasso, ${ }^{3}$ J. D. Joannopoulos, ${ }^{1}$ and Steven G. Johnson ${ }^{1}$ \\ ${ }^{1}$ Center for Materials Science and Engineering, Massachusetts Institute of Technology, Cambridge, MA 02139 \\ ${ }^{2}$ Faculty of Sciences, Department of Physics and Astronomy, Vrije Universiteit Amsterdam, The Netherlands \\ ${ }^{3}$ Department of Physics, Harvard University, Cambridge, MA 02139
}

\begin{abstract}
We present a method of computing Casimir forces for arbitrary geometries, with any desired accuracy, that can directly exploit the efficiency of standard numerical-electromagnetism techniques. Using the simplest possible finite-difference implementation of this approach, we obtain both agreement with past results for cylinder-plate geometries, and also present results for new geometries. In particular, we examine a piston-like problem involving two dielectric and metallic squares sliding between two metallic walls, in two and three dimensions, respectively, and demonstrate non-additive and non-monotonic changes in the force due to these lateral walls.
\end{abstract}

PACS numbers:

Casimir forces arise between macroscopic objects due to changes in the zero-point energy associated with quantum fluctuations of the electromagnetic field [1]. This spectacular effect has been subject to many experimental validations, as reviewed in Ref. 2, All of the experiments reported so far have been based on simple geometries (parallel plates, crossed cylinders, or spheres and plates). For more complex geometries, calculations become extremely cumbersome and often require drastic approximations, a limitation that has hampered experimental and theoretical work beyond the standard geometries.

In this letter, we present a method to compute Casimir forces in arbitrary geometries and materials, with no uncontrolled approximations, that can exploit the efficient solution of well-studied problems in classical computational electromagnetism. Using this method, which we first test for geometries with known solutions, we predict a non-monotonic change in the force arising from lateral side walls in a less-familiar piston-like geometry (Fig. 2). Such a lateral-wall force cannot be predicted by "additive" methods based on proximity-force or other purely two-body-interaction approximations, due to symmetry, and it is difficult to find a simple correction to give a non-monotonic force. We are able to compute forces for both perfect metals and arbitrary dispersive dielectrics, and we also obtain a visual map of the stress tensor that directly depicts the interaction forces between objects.

The Casimir force was originally predicted for parallel metal plates, and the theory was subsequently extended to straighforward formulas for any planar-multilayer dielectric distribution $\varepsilon(x, \omega)$ via the generalized Lifshitz formula 3 . In order to handle more arbitrary geometries, two avenues have been pursued. First, one can employ approximations derived from limits such as that of parallel plates; these methods include the proximity-force approximation (PFA) and its refinements 4], renormalized Casimir-Polder [5] or semi-classical interactions [6], multiple-scattering expansions [7, classical ray optics [8], and various perturbative techniques 9, 10. Such methods, however, involve uncontrolled approximations when applied to arbitrary geometries outside their range of applicability, and have even been observed to give qualitatively incorrect results [11, 12]. Therefore, researchers have instead sought numerical methods applicable to arbitrary geometries that converge to the exact result given sufficient computational resources. One such method uses a path-integral representation for the effective action [13, and has predicted the force between a cylinder and a plane or between corrugated surfaces. Ref. 13 uses a surface parameterization of the fields coupled via vacuum Green's functions, requiring $O\left(N^{2}\right)$ storage and $O\left(N^{3}\right)$ time for $N$ degrees of freedom, making scaling to three dimensions (3d) problematic. Another exact method is the "world-line approach" 12, 14, 15, based on Monte-Carlo path-integral calculations. (The scaling of the world-line method involves a statistical analysis, determined by the relative feature sizes in the geometry, that is beyond the scope of this Letter.) Furthermore, the methods of Ref. 13 and Ref. 14 have currently only been demonstrated for perfect-metallic $z$-invariant structures - in this case, the vector unknowns can be decomposed into TE $(\mathbf{E} \cdot \hat{\mathbf{z}}=0)$ and $\mathrm{TM}(\mathbf{H} \cdot \hat{\mathbf{z}}=0)$ scalar fields with Dirichlet or Neumann boundary conditionsalthough generalizations have been proposed [16. Here, we propose a method based on evaluation of the mean stress tensor via the fluctuation-dissipation theorem, which only involves repeated evaluation of the electromagnetic imaginary-frequency Green's function. For a volume discretization with $N$ degrees of freedom and an efficient iterative solver, this requires $O(N)$ storage and $O\left(N^{2-1 / d}\right)$ time in $d$ dimensions. Furthermore, because evaluation of the Green's function is such a standard problem in classical computational electromagnetism, it will be possible to exploit many developments in fast solvers, such as finite-element, or boundaryelement methods [17. To illustrate the method, our 
initial implementation is based on the simplest-possible finite-difference frequency-domain (FDFD) method, as described below.

As derived by Dzyaloshinskiı et al. [1], the net Casimir force on a body can be expressed as an integral over any closed surface around the body of the mean electromagnetic stress tensor $\left\langle T_{i j}\right\rangle$, integrated over imaginary frequencies $\omega=i w$ :

$$
F_{i}=\int_{0}^{\infty} \frac{\hbar d w}{\pi} \oiiint_{\text {surface }} \sum_{j}\left\langle T_{i j}(\mathbf{r}, i w)\right\rangle d S_{j} .
$$

For a $3 \mathrm{~d} z$-invariant structure, the $z$ integral is replaced by an integral over the corresponding wavevector, resulting in a net force per unit length. The stress tensor is defined as usual by:

$$
\begin{aligned}
& \left\langle T_{i j}(\mathbf{r}, i w)\right\rangle=\left\langle H_{i}(\mathbf{r}) H_{j}(\mathbf{r})\right\rangle-\frac{1}{2} \delta_{i j} \sum_{k}\left\langle H_{k}(\mathbf{r}) H_{k}(\mathbf{r})\right\rangle \\
& +\varepsilon(\mathbf{r}, i w)\left[\left\langle E_{i}(\mathbf{r}) E_{j}(\mathbf{r})\right\rangle-\frac{1}{2} \delta_{i j} \sum_{k}\left\langle E_{k}(\mathbf{r}) E_{k}(\mathbf{r})\right\rangle\right] .
\end{aligned}
$$

The connection to quantum mechanics arises from the correlation functions of the fluctuating fields, such as $\left\langle E_{i} E_{j}\right\rangle$, given via the fluctuation-dissipation theorem in terms of the imaginary- $\omega$ Green's function $G_{i j}\left(i w ; \mathbf{r}-\mathbf{r}^{\prime}\right)$ :

$$
\begin{gathered}
\left\langle E_{i}(\mathbf{r}) E_{j}\left(\mathbf{r}^{\prime}\right)\right\rangle=\frac{w^{2}}{c^{2}} G_{i j}\left(i w ; \mathbf{r}-\mathbf{r}^{\prime}\right) \\
\left\langle H_{i}(\mathbf{r}) H_{j}\left(\mathbf{r}^{\prime}\right)\right\rangle=-(\nabla \times)_{i \ell}\left(\nabla^{\prime} \times\right)_{j m} G_{\ell m}\left(i w ; \mathbf{r}-\mathbf{r}^{\prime}\right),
\end{gathered}
$$

where the Green's function $G_{i j}$ solves the equation:

$$
\left[\nabla \times \nabla \times+\frac{w^{2}}{c^{2}} \varepsilon(\mathbf{r}, i w)\right] \mathbf{G}_{j}\left(i w ; \mathbf{r}-\mathbf{r}^{\prime}\right)=\hat{\mathbf{e}}_{j} \delta\left(\mathbf{r}-\mathbf{r}^{\prime}\right)
$$

for a unit vector $\hat{\mathbf{e}}_{j}$ in the $j$ direction, and obeys the usual boundary conditions on the electric field from classical electromagnetism. (The above expressions are at zero temperature; the nonzero-temperature force is found by changing $\int d w$ in Eq. 1 into a discrete summation [1.) Although the Green's function (and thus $T_{i j}$ ) is formally infinite at $\mathbf{r}=\mathbf{r}^{\prime}$, this divergence is conventionally removed by subtracting the vacuum Green's function; in a numerical method with discretized space, as below, there is no divergence and no additional regularization is required. (The vacuum Green's function gives zero net contribution to the $d \mathbf{S}$ integral, and therefore need not be removed as long as the integrand is finite.)

Historically, this stress-tensor expression was used to derive the standard Lifshitz formula for parallel plates, where $G_{i j}$ is known analytically. However, it also forms an ideal starting point for a computational method, because the Green's function for arbitrary geometries is routinely computed numerically by a variety of techniques [17. Furthermore, the problem actually becomes easier for an imaginary $\omega$. First, for an imaginary $\omega$, the linear operator in Eq. 5 is real-symmetric and positivedefinite for $w \neq 0$, since the dielectric function $\varepsilon(\omega)$ is purely real and positive along the imaginary- $\omega$ axis for physical materials without gain, due to causality. Second, the imaginary- $\omega$ Green's function is exponentially decaying rather than oscillating, leading to a well-behaved nonoscillatory integrand in Eq. 1 .

To illustrate this method, we employed the simplest possible computational technique: we perform a FDFD discretization of Eq. 5 with a staggered Yee grid [18] and periodic boundaries, inverting the linear operator by a conjugate-gradient method. The presence of discontinuous material interfaces degrades second-order finitedifference methods to only first-order accuracy, and the uniform spatial resolution is also suboptimal, but we found FDFD to be nevertheless adequate for small $2 \mathrm{~d}$ geometries. The periodicity leads to artificial "wraparound" forces that decay rapidly with cell size $L$ (at least as $1 / L^{3}$ in $2 \mathrm{~d}$ and $1 / L^{4}$ in $3 \mathrm{~d}$ ); we chose cell sizes large enough to make these contributions negligible $(<1 \%)$.

The computational process is as follows: pick some surface/contour around a given body, evaluate the Green's function for every grid point on this surface in order to compute the surface integral of the stress tensor, which is then integrated over $w$ by adaptive quadrature.

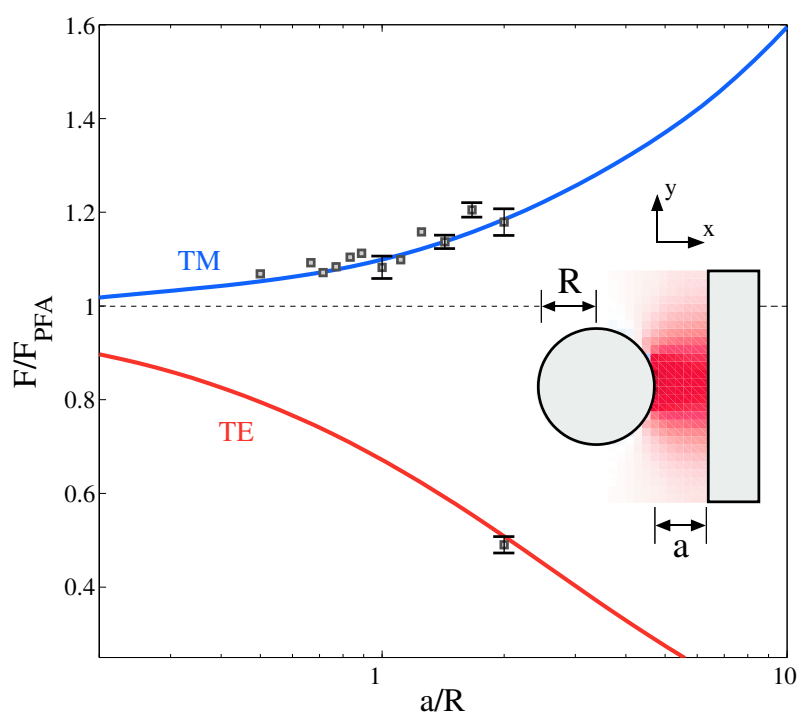

FIG. 1: Casimir force between a radius- $R$ cylinder and a plate (inset), relative to the proximity-force approximation $F_{\mathrm{PFA}}$, vs. normalized separation $a / R$. The solid lines are the Casimir force computed in Ref. 19 for TE (gray) and TM (blue) polarizations, along with results computed by our method with a simple finite-difference discretization (gray squares). Error bars were estimated for some data points by using computations at multiple spatial resolutions. Inset shows interaction stress tensor $\Delta\left\langle T_{x x}\right\rangle$ at a typical imaginary frequency $w=2 \pi c / a$, where red indicates attractive stress.

Before we attempt to study new geometries with our 
method, it is important to check it against known results. The simplest cases, of parallel metallic or dielectric plates, of course match the known result from the Liftshitz formula and are not reproduced here. A more complicated geometry, consisting of a perfect metallic cylinder adjacent to a perfect metallic plate in $3 \mathrm{~d}$, was solved numerically by Ref. [19, to which our results are compared in Fig. 1] Ref. 19 used a specialized FourierBessel basis specific to this cylindrical geometry, which should have exponential (spectral) convergence. Our use of a simple uniform grid was necessarily much less efficient, especially with the first-order accuracy, but was able to match the Ref. 19 results within $\sim 3 \%$ using reasonable computational resources. A simple grid has the advantage of being very general, as illustrated below, but other general bases with much greater efficiency are possible using finite-element or boundary-element methods; the latter, in particular, could use a spectral Fourier basis analogous to Ref. 19 and exploit a fast-multipole or similar $O(N \log N)$ solver technique [17].

Also shown, in the inset of Fig. 1, is a plot of the interaction stress-tensor component $\Delta\left\langle T_{x x}\right\rangle$ at a typical imaginary frequency $w=2 \pi c / a$. By "interaction" stresstensor $\Delta\left\langle T_{i j}\right\rangle$, we mean the total $\left\langle T_{i j}\right\rangle$ of the full geometry minus the sum of the $\left\langle T_{i j}\right\rangle$ 's computed for each body in isolation. Here, the stress tensors of the isolated cylinder and plate have been subtracted, giving us a way to visualize the force due to the interaction. As described below, such stress plots reveal the regions in which two objects most strongly affect one another, and therefore reveal where a change of the geometry would have the most impact. (In contrast, Ref. 12 plots an interactionenergy density that does not directly reveal the force, since the force requires the energy to be differentiated with respect to $a$. For example, Ref. 12 subtracted energy density apparently goes nearly to zero as a metallic surface is approached, whereas the stress tensor cannot since the stress integration surface is arbitrary.)

We now consider a more complicated geometry in which there are interactions between multiple bodies: a 3d "piston"-like structure, shown in the inset of Fig. 2, consisting of two $z$-invariant metal $s \times s$ squares separated by a distance $a$ from one another (here, $s=a$ ) and separated by a distance $h$ from infinite metal plates on either side. We then compute the Casimir force per unit $z$ between the two squares as a function of the separation $h$. The result for perfect conductors is shown in Fig. 2 plotted for the TE and TM polarizations and also showing the total force. (Error bars are not shown because the estimated error is $<1 \%$.) In the limit of $h \rightarrow 0$, this structure approaches the "Casimir piston," which has been solved analytically [20, 21] (and also in $2 \mathrm{~d}$ for the TM polarization [22]). Our results, extrapolated to $h=0$, agree with these results to within $\approx 2 \%$ (although we have computational difficulties for small $h$ due to the high resolution required to resolve a small

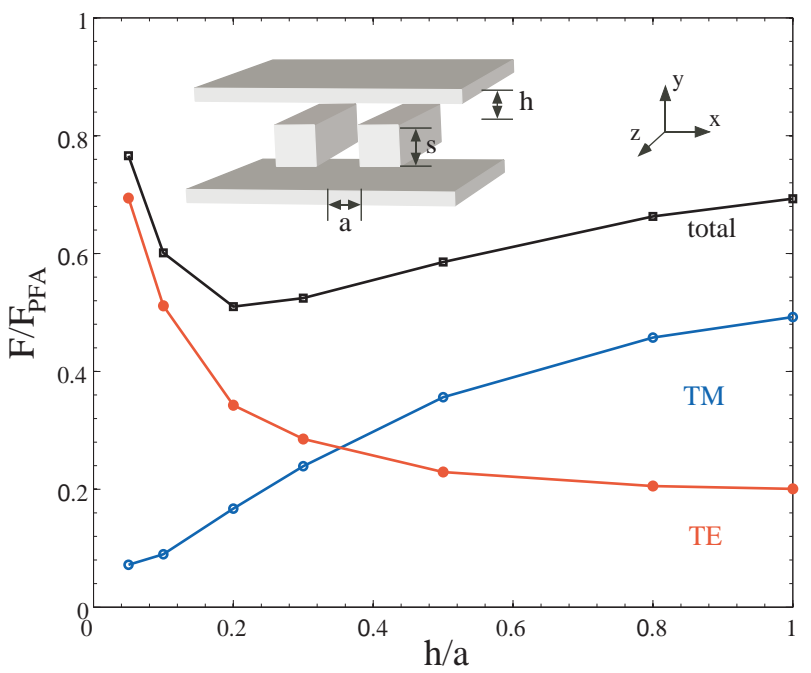

FIG. 2: Casimir force per unit length between metal squares $F / F_{\mathrm{PFA}}$, vs. distance from metal plate $h$ (inset), normalized by the total TE+TM force per unit length obtained using the PFA, $F_{\mathrm{PFA}}=\hbar c s \zeta(3) / 480 \pi a^{4}$. The total force is plotted (black squares) along with the TE (red dots) and TM (blue circles) contributions.

feature in FDFD). For $h>0$, however, the result is surprising in at least two ways. First, the total force is non-monotonic in $h$, due to a competition between the TE and TM contributions to the forces. Second, the $h$ dependence of the force is a lateral effect of the parallel plates on the squares, which would be zero by symmetry in PFA or any other two-body-interaction approximation. Although lateral-wall effects can clearly arise qualitatively in various approximations, such as in ray optics or in PFA restricted to "line-of-sight" interactions, nonmonotonicity is more surprising 24]. Also, in the large- $h$ limit, the force remains different from PFA due to finite- $s$ "edge" effects [12, which are captured by our method.

Our method is also capable, without modification, of handling dielectric materials. This is demonstrated in Fig. 3, where the Casimir force is shown for the case where the squares are made of gold, using the experimental Drude $\varepsilon(\omega)$ from Ref. 23 for a separation $a=1 \mu \mathrm{m}$. Here, our calculation is for a purely $2 \mathrm{~d}$ geometry (equivalently, 3d restricted to $z$-invariant fields/currents), and for comparison we also plot the corresponding $2 \mathrm{~d}$ force for perfect-metal squares (although the two cases are normalized differently as described in the caption). As might be expected, the dielectric squares have a weaker interaction than the perfect-metal squares, but are still non-monotonic. Note also the qualitative similarity between the perfect-metal results of Figs. 2 and 3 reflecting the fact that the force contributions are dominated by the zero-wavevector ( $z$-invariant) fields. Extrapolated to $h=0$, the perfect-metal TM force agrees with the known analytical result 22 to within $\approx 3 \%$. 


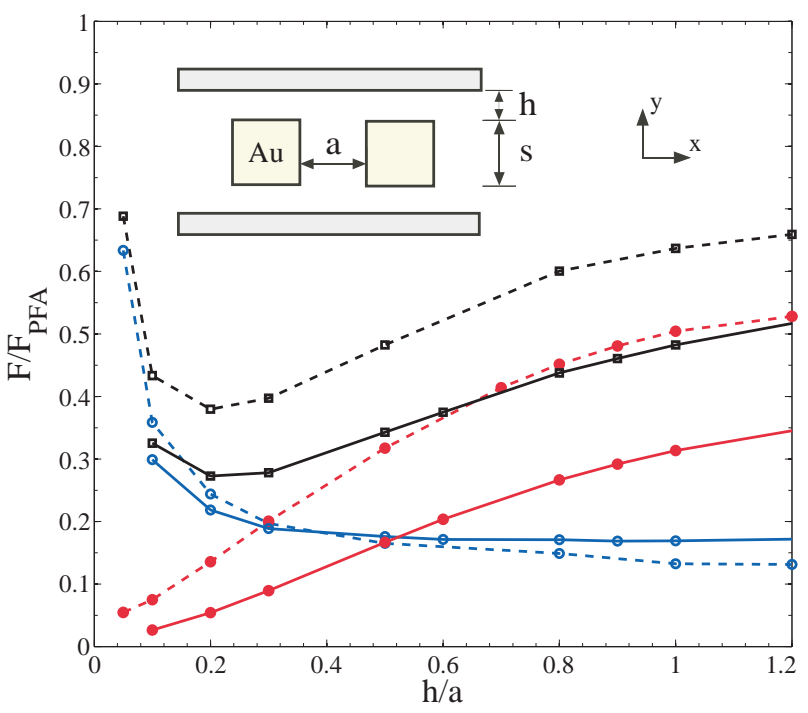

FIG. 3: Solid lines: Casimir force between $2 \mathrm{~d}$ gold squares $F / F_{\mathrm{PFA}}$, vs. distance from metal plate $h$ (inset), using experimental $\varepsilon(\omega)$ [23], normalized by the total force obtained using the PFA. (Here, the PFA force is computed for $x$-infinite gold slabs). The total force is plotted (black squares) along with the TE (red dots) and TM (blue circles) contributions. Dashed lines: force for $2 \mathrm{~d}$ perfect-metal squares, normalized by the perfect-metal PFA force $F_{\mathrm{PFA}}=\hbar \operatorname{cs} \zeta(3) / 8 \pi a^{3}$.

To further explore the source of the $h$-dependence, we plot the TM interaction-stress maps $\Delta\left\langle T_{x x}\right\rangle$ and $\Delta\left\langle T_{x y}\right\rangle$ in Fig. 4, for the 2d perfect-metal squares from Fig. 3 . The stress plots of Fig. 4 are computed at a typical frequency $w=2 \pi c / a$, and for varying distances from the metal plates $(h=0.5,1.0,2.0)$. As shown, the magnitudes of both the $x x(\mathrm{a}-\mathrm{c})$ and $x y(\mathrm{~d}-\mathrm{f})$ components of the stress tensor change dramatically as the metal plates are brought closer to the squares. For example, one change in the force integral comes from $T_{x y}$, which for isolated squares has an asymmetric pattern at the four corners that will contribute to the attractive force, whereas the presence of the plates induces a more symmetric pattern of stresses at the four corners that will have nearly zero integral. This results in a decreasing TM force with decreasing $h$. Because stress maps indicate where bodies interact and with what signs, it may be useful in future work to explore whether they can be used to design unusual behaviors such as non-additive, non-monotonic, or even repulsive forces.

This work was supported in part by the Nanoscale Science and Engineering Center (NSEC) under NSF contract PHY-0117795, by the Materials Research Science and Engineering Center program of the NSF under award DMR-9400334, and by a DOE Comp. Science Grad. Fellowship under grant DE-FG02-97ER25308. D. I. gratefully acknowledges support from the Netherlands Organisation for Scientific Research (NWO), under the IRI Scheme Vernieuwingsimpuls VIDI-680-47-209. (a)

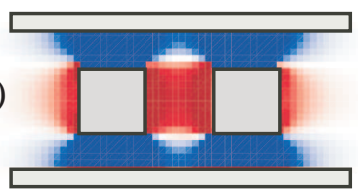

(b)

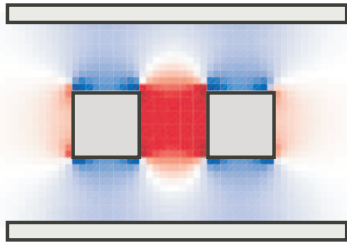

(c)

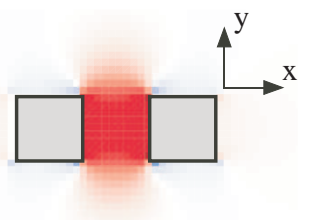

$\Delta \mathrm{T}_{\mathrm{xx}}$ (d)

(e)

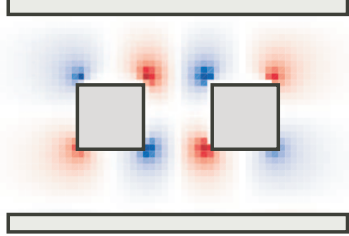

(f)

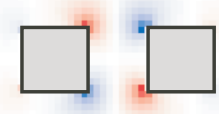

$\Delta \mathrm{T}_{\mathrm{xy}}$

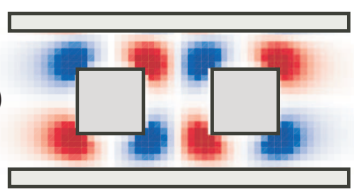

FIG. 4: (a-f): TM stress map of the 2d-analogous geometry of Fig. 2 for various $h$. The intearaction stress tensors $\left\langle T_{x x}\right\rangle$ (left) and $\left\langle T_{x y}\right\rangle$ (right) for: (a),(d): $h=0.5 a$; (b),(e): $h=a$; and (c),(f): $h=2 a$, where blue/white/red $=$ repulsive/zero/attractive.

[1] E. M. Lifshitz and L. P. Pitaevskii, Statistical Physics: Part 2 (Pergamon, Oxford, 1980).

[2] R. Onofrio, New J. Phys. 8, 237 (2006).

[3] M. S. Tomaš, Phys. Rev. A 66, 052103 (2002).

[4] M. Bordag, Phys. Rev. D 73, 125018 (2006).

[5] M. Tajmar, Intl. J. Mod. Phys. C 15, 1387 (2004).

[6] M. Schaden and L. Spruch, Phys. Rev. A 58, 935 (1998).

[7] R. Balian and B. Duplantier, Ann. Phys. 112, 165 (1978).

[8] R. L. Jaffe and A. Scardicchio, Phys. Rev. Lett. 92, 070402 (2004).

[9] T. Emig, A. Hanke, R. Golestanian, and M. Kardar, Phys. Rev. Lett. 87, 260402 (2001).

[10] R. B. Rodrigues, P. A. M. Neto, A. Lambrecht, and S. Reynaud, Phys. Rev. Lett. 96, 100402 (2006).

[11] O. Kenneth, I. Klich, A. Mann, and M. Revzen, Phys. Rev. Lett. 89, 033001 (2002).

[12] H. Gies and K. Klingmuller, Phys. Rev. Lett. 97, 220405 (2006).

[13] R. Büscher and T. Emig, Phys. Rev. Lett. 94, 133901 (2005).

[14] H. Gies, K. Langfeld, and L. Moyaerts, J. High Energy Phys. p. 018 (2003).

[15] H. Gies and K. Klingmuller, Phys. Rev. Lett. 96, 220401 (2006)

[16] T. Emig and R. Büscher, Nucl. Phys. B 696, 468 (2004).

[17] W. C. Chew, J. Jian-Ming, E. Michielssen, and S. Jiming, Fast and Efficient Algorithms in Computational Electromagnetics (Artech, Norwood, MA, 2001).

[18] A. Christ and H. L. Hartnagel, IEEE Trans. Microwave Theory Tech. 35, 688 (1987).

[19] T. Emig, R. L. Jaffe, M. Kardar, and A. Scardicchio, 
Phys. Rev. Lett. 96, 080403 (2006).

[20] M. P. Hertzberg, R. L. Jaffe, M. Kardar, and A. Scardicchio, Phys. Rev. Lett. 95, 250402 (2005).

[21] V. M. Marachevsky, PRD 75, 085019 (2007).

[22] R. M. Cavalcanti, Phys. Rev. D 69, 065015 (2004).
[23] I. Brevik, J. B. Aarseth, J. S. Hoye, and K. A. Milton, Phys. Rev. E 71 (2005).

[24] A publication with R. L. Jaffe is in preparation that shows non-monotonicity arising in ray optics. 